\title{
¿HUBO CONVERGENCIA ECONÓMICA ENTRE LOS PAÍSES EUROPEOS ANTES DE LA I GUERRA MUNDIAL?
}

\author{
DANIEL A. TIRADO FABREGAT \\ JORDI PONS NOVELL \\ Universitat de Barcelona
}

\section{RESUMEN}

En este trabajo se ofrece un resumen de la literatura teórica y empírica sobre la convergencia económica y se presenta una primera caracterización del comportamiento del PIB per cápita relativo en Europa en el período previo a la I Guerra Mundial. Del estudio con datos de corte transversal se deduce el incumplimiento de la hipótesis de $\beta$-convergencia y se verifica la existencia de $\beta$-convergencia a estados estacionarios que son distintos para cada uno de los países. El análisis con datos de serie temporal refuerza el cuadro. Durante el período 1870-1913, sólo los países con niveles de renta per cápita de partida medios lograron acercarse a los niveles de PIB per cápita de los inicialmente más ricos.

\section{ABSTRACT}

This article offers a survey of the theoretical and empirical literature on economic convergence. It also carries out a first attempt to characterize the evolution of relative per capita GDP levels across European countries before IWW (1870-1913). Cross section analysis confirms the inexistence of $\beta$-con-

N. de E.: Fecha de recepción del original: noviembre 1999.

Fecha de la revisión definitiva: diciembre 2000.

* El estudio se ha beneficiado de la financiación ofrecida por el Ministerio de Educación y Cultura a través de los proyectos: PB 98-1187 y SEC 99-0432. Daniel Tirado también ha recibido financiación del Ministerio de Educación y Cultura a través de la ayuda SM1999-0004. Los autores agradecen los comentarios y sugerencias de Joan Ramon Borrell, Alfonso Herranz, Marc Prat, Carles Sudrià y tres evaluadores anónimos. 
vergence but the acomplishment of $\beta$-convergence hipothesis in a model that allows the consideration of different steady-states across countries. Time series analysis confirms that, during the period 1870-1913, only countries with medium per capita GDP levels converged on those having the highest income levels in 1870.

\section{INTRODUCCIÓN}

La mayor parte de la abundante literatura empírica sobre los procesos de convergencia ha analizado, básicamente, el período posterior a 1950. Sin embargo, en los trabajos seminales de Abramovitz (1986) o Baumol (1986) se insistía sobre la necesidad de estudiar la convergencia económica como un proceso de largo plazo. En este sentido, Jeffrey G. Williamson ha sostenido que es conveniente analizar estos procesos en un ámbito temporal que debería arrancar en la segunda mitad del siglo XIX. En particular, ha resaltado que el primer gran período en el que la convergencia económica caracterizó el comportamiento agregado de la muestra de países que compondrían el llamado club OCDE fue el que va entre 1870 y $1913^{1}$.

Centrándose en este período, el análisis de los determinantes de la convergencia entre las economías atlánticas ha servido para mostrar la relevancia de factores poco destacados en los estudios referidos al período posterior a la II Guerra Mundial. Por una parte, un grupo de trabajos ha demostrado la importancia de la globalización de los mercados de factores en la comprensión de la convergencia económica ${ }^{2}$. A la vez, se ha señalado que el catch-up tecnológico, entendido como acercamiento en la productividad del trabajo en los sectores industriales, no parece ser una pieza relevante en la realización del proceso ${ }^{3}$. En definitiva, el cambio en la estructura del producto, favorecido por el flujo de factores (trabajo y capital) y bienes que caracterizó al periodo, debe ser considerado el elemento clave en la comprensión de la dinámica de crecimiento en este período ${ }^{4}$.

${ }^{1}$ Williamson (1996), pp. 277-285. Para el período 1870-1913, los países que componen este club OCDE (también llamado Economía Atlántica) serían Dinamarca, Finlandia, Noruega, Suecia, Italia, Portugal, España, Irlanda, Bélgica, Francia, Alemania, Gran Bretaña, Holanda, Argentina, Australia, Canadá y Estados Unidos.

2 Williamson (1996), pp. 288-295, O'Rourke y Williamson (1997), pp. 168-172.

${ }^{3}$ Broadberry (1996), pp. 335-341.

${ }^{4}$ Williamson (1996), p. 278, y Broadberry (1996), p. 343. Esta conclusión está directamente relacionada con la tesis que, para el análisis de la evolución de la economia británica, ha sido sostenida por N. F. R. Crafts. Una sintesis en Crafts (1998). 
Sin embargo, cuando se ha restringido la muestra seleccionada a los países europeos, los resultados han sido menos claros. En este sentido, Gabriel Tortella ha destacado la existencia de una pauta de comportamiento en los países más periféricos de Europa que sería de divergencia durante el siglo XIX y convergencia en el $\mathrm{xx}^{5}$. El propio Jeffrey Williamson, aun manteniendo que la convergencia caracterizó el comportamiento de los países europeos durante estos años, ha reconocido que ésta tuvo una envergadura modesta en términos agregados, ya que los casos más exitosos (países escandinavos) fueron compensados por fracasos significativos (España y Portugal). En cualquier caso, recomienda Williamson, la experiencia europea merece una mirada más atenta ${ }^{6}$.

Por ello, a lo largo de estas páginas, presentaremos una primera caracterización del comportamiento del PIB per cápita relativo en Europa en el período previo a la I Guerra Mundial. En particular, trataremos de identificar la existencia de patrones de convergencia entre los Estados europeos $y$, en última instancia, analizaremos si éstos favorecieron la reducción de las divergencias existentes entre las distintas economías o si, por contra, limitaron esta posibilidad a algunos pocos países. Apoyándonos en esta evidencia, trataremos de avanzar hipótesis razonadas sobre los determinantes de la dinámica de crecimiento comparado de los países europeos durante estos años que puedan servir para completar el diagnóstico sugerido por los trabajos reseñados.

El trabajo se organiza de la siguiente manera. En primer lugar, en el punto dos, presentaremos el marco teórico. A continuación, en el punto tres, ofreceremos algunos conceptos y métodos empleados habitualmente en el análisis empírico de los procesos de convergencia. El punto cuatro servirá para presentar la base de datos sobre la que se construye el estudio. Posteriormente, el punto cinco ofrecerá una primera aproximación a la dinámica del PIB per cápita relativo en Europa durante los años 1870-1913 utilizando dos aproximaciones empíricas al estudio de la convergencia: la que se deriva del análisis de datos de corte transversal y la ofrecida por

Tortella (1994), p. 1. Entre ellos se situarían Italia, España y Portugal.

${ }^{6}$ En este sentido, cabe señalar la práctica inexistencia de estudios empíricos sobre la convergencia entre los Estados europeos durante el período previo a la Gran Guerra Entre los que centran su análisis en este periodo, la mayor parte analiza la evolución relativa de las economías europeas en muestras que incluyen un número más amplio de países (éste sería el caso de muchos de los trabajos publicados por Jeffrey Williamson y su grupo de colaboradores, citados en la nota 2). Un precedente cercano al trabajo que aquí se presenta sería Prados, Dabán y Sanz (1993), pp. 3-12. 
el estudio de datos de serie temporal. Unos comentarios finales sintetizan las principales conclusiones que se derivan del análisis realizado.

\section{EL MARCO TEÓRICO ${ }^{7}$}

El estudio de la evolución relativa de la renta per cápita entre distintas economías es un elemento de importancia clave en la literatura del crecimiento económico. En ésta, la predicción que se establece tanto sobre la viabilidad del crecimiento económico sostenido como sobre la evolución de las divergencias internacionales en renta per cápita, depende básicamente de dos supuestos tecnológicos. La existencia o no de rendimientos decrecientes en el uso de los factores, incluido el conocimiento técnico, y la consideración de si éste es o no un bien público entre los países.

Dentro de esta vasta literatura, la versión más sencilla de los modelos neoclásicos apuesta por la existencia de rendimientos decrecientes en el uso de los factores y por el libre acceso de todas las economías a un nivel de desarrollo tecnológico común. Por ello, este tipo de modelos predice que el crecimiento de la renta per cápita no es sostenible en el largo plazo. Por contra, ofrecen implicaciones positivas sobre la evolución tendencial de los diferenciales de renta existentes entre las economías ${ }^{8}$.

Sin progreso técnico, los rendimientos decrecientes en el uso del capital suponen que su producto marginal decrece en la medida que una economía alcanza un nivel de renta y un stock de capital per cápita más alto. Con ello, se reducen los incentivos al ahorro y la contribución al crecimiento de la renta de un volumen dado de inversión. Con esta lógica interna, de este tipo de modelizaciones emana la hipótesis de convergencia a largo plazo en los niveles de renta: los países más pobres tendrán mayores incentivos a ahorrar y mayores tasas de crecimiento del producto ante inversiones similares. Por lo tanto, estos países tenderán a reducir la distancia que los separa de los países más ricos. Además, en este marco teórico, las consideraciones de economía abierta refuerzan la predicción. Los flujos internacionales de bienes y factores contribuyen a la igualación internacional de los precios y con ello al cumplimiento de la predicción de convergencia en los niveles de renta per cápita.

${ }^{7}$ Este apartado es deudor de las síntesis sobre la conceptualización teórica de la convergencia ofrecidas en Galor (1996), pp. 1056-1061, Sala-i-Martin (1996), pp. 1020-1028, y De la Fuente (1997), pp. 30-36.

${ }^{8}$ El punto de partida de esta conceptualización se encuentra en Solow (1956). 
La introducción de la posibilidad de progreso técnico exógeno facilita la explicación del crecimiento de largo plazo y no modifica las predicciones en términos de convergencia, siempre y cuando consideremos a la tecnología como un bien público, al que los países tienen libre acceso. En este sentido, aparece un nuevo elemento impulsor de la convergencia, el catch-up tecnológico?.

Sin embargo, los trabajos empíricos sobre la evolución de las disparidades de renta en el largo plazo no han servido para confirmar las predicciones que emanan de las modelizaciones neoclásicas del crecimiento interpretadas de una forma extrema. Ésta es una de las razones que estimuló la aparición de un amplio y heterogéneo grupo de trabajos que podríamos encuadrar en la acepción de literatura del crecimiento endógeno y que ha ofrecido una alternativa a las aproximaciones neoclásicas al concepto de convergencia ${ }^{10}$. Estos estudios han analizado la dinámica de crecimiento de las economías en caso de aceptarse otros supuestos de partida distintos a los del modelo neoclásico; recordemos, rendimientos decrecientes y determinación exógena del progreso técnico. Han venido a demostrar que la aceptación de rendimientos crecientes en la función de producción agregada permite invertir las predicciones que sobre evolución de las disparidades internacionales de renta genera la visión neoclásica. En esencia, la nueva asunción nos llevaría a argumentar que la tasa de crecimiento debe crecer con el tiempo y con el nivel de renta. Con ello, las divergencias iniciales tenderían a incrementarse.

Versiones menos extremas en este cuerpo de la literatura explican la sostenibilidad del crecimiento en formulaciones en las que el progreso técnico es determinado de forma endógena mediante las decisiones privadas de inversión en capital humano o tecnológico siempre que este tipo de actividades no estén sujetas a rendimientos decrecientes. Estos modelos predicen la existencia de divergencias permanentes en los niveles de renta de los distintos países y que éstas pueden deberse a diferencias en las políticas económicas implementadas, en las dotaciones iniciales de factores o en el tamaño de mercado.

Este tipo de predicciones parece acercarse más a los hechos estilizados del crecimiento económico contemporáneo. Sin embargo, una visión más detenida de los modelos neoclásicos anuncia que sus predicciones pueden

Sobre el particular ver Abramovitz (1986), pp. 386-390.

${ }_{10}$ Estos trabajos se inspiran en ideas popularizadas en aportaciones clásicas como las de Arrow (1962), Shell (1967). Véase Romer (1986), Lucas (1988) o Grossman y Helpman (1991). 
no diferir tanto de las anunciadas por la literatura del crecimiento endógeno. La contrarrevolución neoclásica ha remarcado que el modelo inicial de Solow sólo predice la tendencia a la reducción de las diferencias internacionales de renta en determinadas condiciones. En realidad, en el mundo neoclásico, los niveles de renta de estado estacionario son una función de las tasas de inversión y de las de crecimiento de la población y éstas pueden diferir entre economías.

Además, aunque el libre acceso a la tecnología abra una oportunidad de catch-up tecnológico que acelere la convergencia en renta de los países más pobres, la existencia de este potencial no asegura su realización. Sólo en caso de que éstos dispongan de las social capabilities que les permitan absorber las nuevas tecnologías y transformarlas de la forma más adecuada a sus necesidades, el retraso tecnológico se plasmará en un proceso de convergencia más pronunciado. Por ello, las divergencias iniciales en dotación de mano de obra cualificada o de personal científico o técnico pueden ser relevantes a la hora de entender la no reducción en la dispersión de la renta entre los países.

Así pues, la ausencia de convergencia entre las economías contemporáneas puede quedar explicada en las modelizaciones neoclásicas, bien porque los países carecen de social capabilities, bien porque no operan en marcos institucionales que favorezcan los flujos internacionales de bienes y factores, bien porque converjan a estados estacionarios que son distintos entre sí. Este sería el concepto de convergencia condicionada que ha alcanzado un elevado grado de consenso entre la profesión.

No obstante, un grupo de autores ha venido a remarcar la existencia de un hecho estilizado más al que debe dar respuesta nuestro marco teórico del crecimiento. En el análisis empírico de la evolución de las economías europeas en el período posterior a la II Guerra Mundial se contrasta la tendencia a que, con independencia de la reducción o no de la dispersión en los niveles renta, el hecho que caracteriza a la evolución de los diferenciales de renta es el acercamiento tendencial de los paises hacia clusters, niveles de renta comunes, que difieren entre distintos grupos de países ${ }^{11}$.

La literatura teórica de tradición neoclásica también ha dado respuesta a esta evidencia ${ }^{12}$. La modelización neoclásica, basada en la aceptación de tecnología de libre acceso y de rendimientos decrecientes en el uso de los factores puede ofrecer respuesta a este tipo de evidencia siempre

${ }^{11}$ Los trabajos de Quah (1996a) o (1997) ofrecen evidencia sobre la formación de clusters o clubs de convergencia.

${ }_{12}$ Un resumen de la misma se encuentra en Galor (1996), p. 1061. 
que se atienda a que puede predecir la existencia de más de un equilibrio de largo plazo estable en la trayectoria de crecimiento de un país. Si esto es así, paises con elementos fundamentales (crecimiento de la población y tasas de inversión), capacidades sociales y entornos institucionales similares pueden converger, verse atraídos, a niveles de renta que sólo son iguales si lo son sus condiciones de partida.

Estas condiciones iniciales pueden estar relacionadas con el nivel de renta per cápita de partida, la distribución inicial del ingreso ${ }^{13}$, la distribución de factores de producción como el capital humano ${ }^{14} \mathrm{o}$ el tamaño de mercado ${ }^{15}$ en marcos teóricos neoclásicos que consideran la heterogeneidad de comportamiento de los agentes, la aparición de rendimientos públicos crecientes en la acumulación de capital humano, o la existencia de complementariedades sectoriales y tecnológicas en presencia de estructuras de mercados no competitivas.

\section{DEFINICIÓN Y CONTRASTES EMPÍRICOS DE LA CONVERGENCIA}

\subsection{Consideraciones previas}

Los trabajos empíricos en el ámbito del crecimiento y de la convergencia económica se han efectuado tanto a partir de datos temporales como transversales. Sin embargo, las definiciones de convergencia implícitas en ambos enfoques son distintas ${ }^{16}$. Los trabajos con datos transversales se han centrado en el estudio de la transición de las distintas economías al estado de equilibrio. En este enfoque se dice que existe convergencia $\beta$ si se encuentra una relación negativa entre la tasa de crecimiento de la renta per cápita y el nivel de renta inicial, es decir, si las economías pobres tienden a crecer más rápidamente que las ricas. Por otra parte, existe convergencia $\sigma$ si la dispersión de la renta real per cápita entre grupos de economías tiende a reducirse en el tiempo.

Además, como hemos señalado, a principios de los años noventa una serie de trabajos empíricos argumentaron que el modelo neoclásico no

${ }^{13}$ Por ejemplo, se ofrecen modelizaciones de este tipo en Galor y Zeira (1993) o Durlauf (1996).

${ }^{14}$ Azariadis y Drazen (1990).

is Murphy, Schleifer y Vishny (1989).

${ }^{16}$ En Bernard y Durlauf (1996) se define formalmente el concepto de convergencia implícito en ambos enfoques. 
predecía de por sí la convergencia económica y concluían que sólo en el caso de que las diferentes economías compartieran las mismas preferencias y la misma tecnología, el modelo neoclásico predeciría la convergencia. Estos autores desarrollaron, por tanto, el concepto de convergencia condicionada o relativa para contraponerlo al de convergencia absoluta ${ }^{17}$. El concepto de convergencia condicionada permitió conciliar el modelo de crecimiento neoclásico con el análisis empírico de la convergencia con datos de corte transversal y posibilitó encontrar evidencia favorable a la hipótesis de convergencia en los estudios empíricos.

En los últimos años se ha puesto en duda la validez de esta metodología ${ }^{18}$. Las principales críticas que le han sido formuladas son que considera una especie de economía representativa al utilizar conjuntamente datos de distintos territorios, su pretensión de explicar la distribución transversal de la renta mediante el uso de dos únicos estadísticos $(\sigma$ y $\beta)$, el hecho de recoger el componente permanente de la renta de cada economía mediante una tendencia lineal determinista y, por último, el estar sometida a la denominada falacia de Galton, que sugiere que la presencia de una relación negativa entre el nivel de renta inicial y su tasa de crecimiento es compatible con la ausencia de convergencia.

Con el objetivo de superar estas limitaciones del análisis empírico con datos de corte transversal se han desarrollado diferentes metodologías, entre las que destaca el estudio de series temporales ${ }^{19}$. En este enfoque se puede distinguir entre los conceptos de catching-up y de convergencia absoluta. Un proceso de catching-up se relaciona con la tendencia a la disminución, en el transcurso del tiempo, de las diferencias entre las series consideradas, pero con la persistencia de distintos estados estacionarios ${ }^{20}$. En cambio, el concepto de convergencia absoluta supone la desaparición con el tiempo de las diferencias de renta y representa, en consecuencia, una versión más estricta de la convergencia ${ }^{21}$.

17 Barro (1991), Barro y Sala-i-Martin (1990) y Mankiw, Romer y Weil (1992). Para el desarrollo de esta literatura empírica fue clave la publicación de una amplia base de datos como la ofrecida en Summers y Heston (1991).

${ }_{18}$ Básicamente a partir de los trabajos de Quah (1993a), (1993b), (1996a), (1996b) y (1997).

19 Otra posibilidad, que no ha sido utilizada en este trabajo aunque se contempla como una línea futura de investigación, se basa en considerar la literatura relacionada con los clubs de convergencia propuesta por Quah.

${ }^{20}$ El estado estacionario es la renta per cápita esperada del país considerado, que depende de sus características sociales, económicas y estructurales.

${ }^{21}$ Entre los trabajos empíricos desarrollados a partir de este enfoque se pueden destacar 


\subsection{Corte transversal: convergencia $\sigma, \beta$ y condicionada}

El concepto de $\sigma$-convergencia hace referencia a la tendencia a la reducción de las diferencias de renta existentes entre los países. Si las economías convergen hacia equilibrios de largo plazo similares, el estudio empírico de economías que caminan hacia este punto debería permitirnos contrastar la reducción en la dispersión existente entre sus niveles de renta.

Sin embargo, esta vía empírica de contraste de la convergencia absoluta se enfrenta a una severa limitación. Incluso en el caso de cumplimiento del modelo neoclásico más sencillo, las divergencias en renta entre economías pueden ampliarse a lo largo del tiempo, no por el incumplimiento de las predicciones neoclásicas, sino por la convivencia de shocks de corto plazo que las alejen de su senda tendencial.

Por ello, la investigación empirica ha hecho hincapié en el contraste de otra definición de convergencia, la de $\beta$-convergencia. Ésta hace referencia a la tendencia de los países pobres a crecer a un ritmo superior a los ricos. En el contraste empírico de esta noción, los investigadores han tratado de comprobar el sostenimiento o no de relaciones como las presentadas en la ecuación (1) ${ }^{22}$ en muestras de sección cruzada, o de sección cruzada combinadas con datos seriados, de un conjunto de economías:

$$
\log \left(y_{i, t}+T / y_{i, t}\right) / T=\alpha-\beta \log \left(y_{i, t}\right)+u_{i, t}
$$

Esta ecuación es deducible de un modelo de crecimiento económico neoclásico de corte estructural y nos muestra cómo la tasa de crecimiento está inversamente relacionada con el nivel de renta per cápita de partida. El contraste empírico de la hipótesis de $\beta$-convergencia se centra en la determinación del poder explicativo de esta forma funcional y en el valor y significatividad del parámetro $\beta$. Si éste es positivo y acotado entre 0 y 1 , la evidencia nos mostraría que los países inicialmente más pobres tienden a crecer de forma más rápida que los más ricos. A la vez, ésta es una condición necesaria, aunque no suficiente, para la reducción tendencial en los diferenciales de renta entre países. Además, el valor del parámetro informa de la velocidad a la que se produce el proceso.

los de Carlino y Mills (1993), Bernard y Durlauf (1995) y Pallardó y Esteve (1997). Por otra parte, es fundamental la aportación de Oxley y Greasley (1995), puesto que introduce la posibilidad de incorporar cambios estructurales al analizar la convergencia entre pares de economías.

${ }^{22}$ En particular, las formas funcionales propuestas son las sugeridas en Barro y Sala-i-Martin (1990) y reproducidas en Sala-i-Martin (1996), pp. 1020 y 1027. 
Sin embargo, los trabajos empíricos sobre grupos heterogéneos de países han venido a demostrar que, muchas veces, el parámetro no toma el signo esperado o presenta valores muy reducidos, lo que nos ilustraría sobre una gran lentitud en los procesos de convergencia. Además, en muchos casos, el poder explicativo de la forma estimada es muy reducido. La teoría expuesta en el punto dos nos ofrece una respuesta a estos resultados. La hipótesis que puede estar cumpliéndose no es la de convergencia absoluta, sino la de convergencia condicionada. Es decir, el crecimiento de los países está inversamente relacionado con su distancia a los niveles de renta de estado estacionario, pero éstos pueden diferir entre economías.

El contraste empírico de esta hipótesis descansa habitualmente en la introducción, en la ecuación inicial, de variables económicas que puedan captar estos diferenciales en los niveles de renta de estado estacionario $o$, en caso de no ser posible, de variables ficticias que nos informen de la significatividad de los mismos.

$$
\log \left(\mathrm{y}_{\mathrm{i}, \mathrm{t}}+\mathrm{T} / \mathrm{y}_{\mathrm{i}, \mathrm{t}}\right) / \mathrm{T}=\alpha-\beta \log \left(\mathrm{y}_{\mathrm{i}, \mathrm{t}}\right)+\psi \mathrm{X}_{\mathrm{i}, \mathrm{t}}+\mathrm{u}_{\mathrm{i}, \mathrm{t}}
$$

En (2), $X_{i, t}$ es el vector que agrupa las variables fundamentales explicativas de las diferencias en el estado estacionario o, en su caso, las variables ficticias encargadas de recoger estos diferenciales. En este caso podemos controlar por la existencia de $\beta$-convergencia condicionada observando el valor y significatividad de $\beta$ y de la variables incluidas en el vector $X$. Además, en caso de captar los diferentes estados estacionarios a partir de variables ficticias, sus valores estimados y su significatividad nos informarán de la persistencia de los diferenciales y de su magnitud. Este proceder equivale, como se verá posteriormente, a la estimación de un modelo de «efectos fijos». Estos efectos, referidos a cada uno de los países estudiados, pueden ser positivos, si el crecimiento de la renta es superior al que cabría esperar teóricamente; o negativos, si el incremento de la renta es inferior a la esperada debido a la presencia de elementos que lo impiden o retrasan ${ }^{23}$.

\subsection{Series temporales: convergencia a largo plazo, catching-up y puntos de ruptura en las series}

La utilización del enfoque basado en las series temporales requiere diferenciar claramente entre los conceptos de catching-up y convergencia

${ }^{23}$ Una explicación sumamente intuitiva de este concepto se puede encontrar en Cuadrado, Mancha y Garrido (1998). 
absoluta presentados anteriormente ${ }^{24}$. Dos economías convergen en términos de catching-up si la diferencia en el producto per cápita entre ambas se estrecha a lo largo del tiempo. El concepto de convergencia a largo plazo es más estricto que el anterior, puesto que se espera que los niveles de producto per cápita en ambas economías se igualen en un determinado momento del tiempo ${ }^{25}$.

Los resultados encontrados en algunos trabajos empíricos, en el sentido de que la evidencia obtenida mediante los contrastes tradicionales de raíces unitarias puede quedar invalidada al estar estos tests sesgados hacia la aceptación espúrea de raíces unitarias en las series analizadas, provocaron la necesidad de incorporar la posible presencia de puntos de ruptura en las series históricas ${ }^{26}$.

En nuestro análisis se estudia la presencia de convergencia a largo plazo y catching-up considerando la opción de que las series contengan rupturas ${ }^{27}$. Para ello se proponen un conjunto de modelos alternativos que contemplan diversas posibilidades para introducir estos cambios estructurales. Además, no se predetermina previamente el punto de ruptura de las series, sino que se utiliza un procedimiento que endogeneiza su búsqueda ${ }^{28}$.

Se utilizan dos tipos de modelos para considerar la posibilidad de que las series contengan rupturas. En primer lugar, los denominados additive outlier models (AOM), cuya contrastación se realiza en dos etapas y se corresponden con la presencia de cambios instantáneos en la serie. En segundo lugar, los innovational outlier models (IOM), que permiten contrastar la presencia de raíces unitarias en una sola etapa y detectan cambios graduales en la serie. El análisis realizado permite, a la vez, distinguir entre un cambio de nivel de la serie, un cambio de tendencia y ambos simultáneamente.

La ecuación utilizada en el caso de un cambio en el nivel es la siguiente:

${ }^{24}$ En Bernard y Durlauf (1996), pp. 165-166, se puede encontrar una presentación más formalizada de estos conceptos.

25 Para que se pueda hablar de un proceso de catching-up entre dos economías es preciso que exista una relación de cointegración estocástica entre ambas, y para que se produzca un proceso de convergencia a largo plazo es indispensable una relación de cointegración tanto estocástica como determinista entre las series analizadas.

${ }^{26}$ Entre estos trabajos pueden citarse los de Perron (1989), Rappoport y Reichlin (1989) y Banerjee, Lumsdaine y Stock (1992). También se puede consultar la reciente aportación de Ben-David y Papell (2000).

${ }^{27}$ En la línea, como se citaba anteriormente, del trabajo de Oxley y Greasley (1995), o el más reciente de Pallardó y Esteve (1997).

${ }^{28}$ Zivot y Andrews (1992), Perron (1994) y Ben-David y Papell (2000). 


$$
y_{t}=\mu+\gamma D U_{t}+\beta t+\delta D(T B)_{t}+\alpha y_{t-1}+\Sigma_{j=1}^{k} c_{j} \Delta y_{t-j}+\varepsilon_{t}
$$

que se corresponde con los modelos AOM-I e IOM-I, aunque en este trabajo únicamente se ha contrastado el cambio en el nivel de la serie a partir de la segunda opción, es decir, la que contempla un cambio gradual en la serie mediante un contraste en una única etapa.

Al contemplar un cambio en la tendencia la expresión usada es:

$$
y_{t}=\mu+\beta t+\theta D T_{t}^{*}+\alpha y_{t-1}+\Sigma_{j=1}^{k} c_{j} \Delta y_{t-j}+\varepsilon_{t}
$$

especificación correspondiente al modelo AOM-II, que permite contrastar en una etapa un cambio instantáneo en la pendiente de la serie. Por otra parte, debe destacarse que el modelo IOM-II (cambio gradual en la tendencia contrastado en dos etapas) no ha sido resuelto en la literatura teórica.

Finalmente, el cambio en nivel y tendencia se puede contrastar a partir de la ecuación:

$$
y_{t}=\mu+\gamma D U_{t}+\beta t+\theta D T_{t}^{*}+\delta D(T B)_{t}+\alpha y_{t-1}+\Sigma_{j=1}^{k} c_{j} \Delta y_{t-j}+\varepsilon_{t}
$$

relacionada con los modelos AOM-III e IOM-III, aunque en nuestro trabajo únicamente se ha utilizado la segunda opción para contrastar los cambios en nivel y tendencia.

En estos modelos, $y_{t}$ es la variable analizada ${ }^{29} ; \mathrm{D}(\mathrm{TB})_{t}, \mathrm{DU}_{\mathrm{t}}$ y $\mathrm{DT}_{\mathrm{t}}{ }^{*}$ son variables ficticias que tratan de recoger, respectivamente, un año anómalo en la serie, una ruptura en el nivel y un cambio en la tendencia. Estas variables se definen de la siguiente manera: $D(T B)_{t}=1$ si $t=T B$,

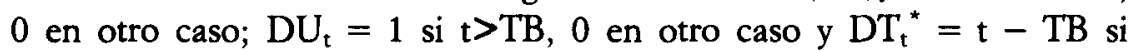
$\mathrm{t}>\mathrm{TB}, 0$ en otro caso; siendo TB el punto de ruptura ${ }^{30}$.

* Es decir, la diferencia en renta per cápita entre pares de economías.

${ }^{30}$ El punto de ruptura para cada una de las series se determina seleccionando el valor de TB (punto de ruptura) que maximiza el estadístico $t$ de Dickey y Fuller asociado al parámetro $\alpha$. El valor de $\mathrm{k}$ se selecciona fijando un valor superior del mismo a priori. En el caso de que el último retardo sea significativo, éste será el valor seleccionado. En caso contrario, se reduce el valor de $\mathrm{k}$ en una unidad hasta que el último retardo sea significativo. En el caso de que ningún retardo sea significativo se establece que $\mathbf{k}=0$. El valor inicial de $\mathrm{k}$ se fija habitualmente en 8 y el criterio de significación del estadístico $\mathrm{t}$ del último retardo se fija en 1,6, que se corresponde con un nivel aproximado de significación del 10 por 100 . 


\section{LA BASE DE DATOS}

El trabajo empírico se basa en la última versión publicada de los datos de PIB per cápita elaborados por Maddison ${ }^{31}$. Las primeras versiones del trabajo del historiador de Newcastle sirvieron para cimentar trabajos como los de Baumol (1986), De Long (1988), Baumol y Wolf (1988), Prados, Dabán y Sanz (1993), Broadberry (1996) o Sala-i-Martin (1996) ${ }^{32}$. Su actualización mejora la calidad de la base de datos inicial y amplía su cobertura con información de un mayor número de países. Por ello, sigue siendo la base de datos utilizada por los estudios empíricos sobre la convergencia entre países que toman como punto de partida la distribución del PIB per cápita a mediados del siglo XIX ${ }^{33}$.

Los valores ofrecidos por Maddison nos permiten contar con la serie anual de PIB per cápita de 12 Estados europeos en el período 1870-1913: Alemania, Austria, Bélgica, Dinamarca, España, Finlandia, Francia, Gran Bretaña, Holanda, Italia, Noruega y Suecia. En nuestro caso hemos ampliado la muestra acudiendo a trabajos adicionales. En este sentido, hemos aprovechado la reciente estimación de la evolución del PNB portugués ofrecida en Bardini, Carreras y Lains (1995) para, utilizando los niveles de PIB per cápita en 1870 y en 1913 ofrecidos por Maddison, construir una serie de PIB per cápita portugués entre 1870 y 1913. Además, hemos sustituido la serie de PIB per cápita español ofrecida por Maddison, por la estimación reciente de Prados de la Escosura (1995). Por lo tanto, nuestra base de datos es un panel con 13 países y 44 observaciones de renta para cada uno de ellos.

La elección de la base de datos no está exenta de problemas. Por una parte, nos obliga a trabajar con datos de PIB per cápita, ya que no disponemos de cifras de PIB por activo empleado, mejor indicador de productividad que el aquí utilizado, para un grupo tan amplio de países. Además, los datos están valorados en dólares PPA de 1990. Es decir, se utiliza para la comparación una cesta de bienes y unos precios relativos fijados en la actualidad. Con ello, como han denunciado Prados de la Escosura y Sanz (1998), las estimaciones retrospectivas están sometidas a sesgos generados por cambios importantes en los precios relativos. Ade-

31 Nos referimos a Maddison (1995).

32 Las primeras versiones tienen su origen en el trabajo publicado en 1982 e incluyen la reelaboración ofrecida en 1991. Maddison (1982) y (1991).

${ }^{33}$ Entre ellos podemos hacer referencia a Taylor (1999). 
más, debe tenerse en cuenta que la calidad de la información de base utilizada para construir las series no es la misma para todos los países.

No obstante, entendemos que es la mejor de las opciones posibles dadas las alternativas existentes. En este sentido, Williamson (1995) ofrece una base de datos de salarios reales para muchos de los países analizados que trataremos de estudiar en próximos trabajos. En cualquier caso, tampoco está exenta de limitaciones. Por una parte, los salarios considerados en cada uno de los países tiene una cobertura muy desigual. Por otra, su utilización nos obligaría a reducir el número de países analizados ${ }^{34}$.

\section{ANÁLISIS EMPÍRICO DE LA CONVERGENCIA}

\subsection{Una primera aproximación a la $\sigma$ y $\beta$-convergencia: el análisis de corte transversal}

La primera aproximación a la $\sigma$-convergencia se presenta en el Gráfico 1, donde se ofrece la evolución de un indicador de dispersión de la muestra, la desviación estándar. En términos generales, podemos afirmar que su evolución no apunta hacia una reducción de la dispersión media del PIB per cápita a lo largo del período. Observando con detalle la evolución del indicador, podríamos señalar la existencia de un cambio en su comportamiento durante estos años. La dispersión de la muestra aumenta hasta mediados de la década de los noventa, y comienza a reducirse, de forma tendencial, desde entonces y hasta 1913.

Sin embargo, algunos trabajos han subrayado la posibilidad de que la predicción neoclásica de la $\sigma$-convergencia estuviera vinculada a la evolución relativa de países con características estructurales comunes. Es decir, que sólo pudiera contrastarse en grupos homogéneos de economías. Siguiendo esta hipótesis de trabajo hemos dividido la muestra en tres bloques de países, atendiendo a su posición de partida. En una primera aproximación hemos separado los países con un PIB per cápita situado entre el 70 y el 90 por 100 de la media muestral (Países pobres: Italia, Noruega, Finlandia, España y Portugal), los que parten de una posición intermedia dentro de la muestra, entre el 90 por 100 y el 110 por 100 (países de renta per cápita media: Austria, Dinamarca, Francia, Alemania y Suecia)

${ }^{34}$ Un comentario crítico sobre la base de datos utilizada en estas páginas y las alternativas existentes se puede encontrar en Broadberry (1996), pp. 341-343. 


\section{GRÁFICO 1}

Convergencia o: $1870-1913$

(desviación estándar PIB per cápita)

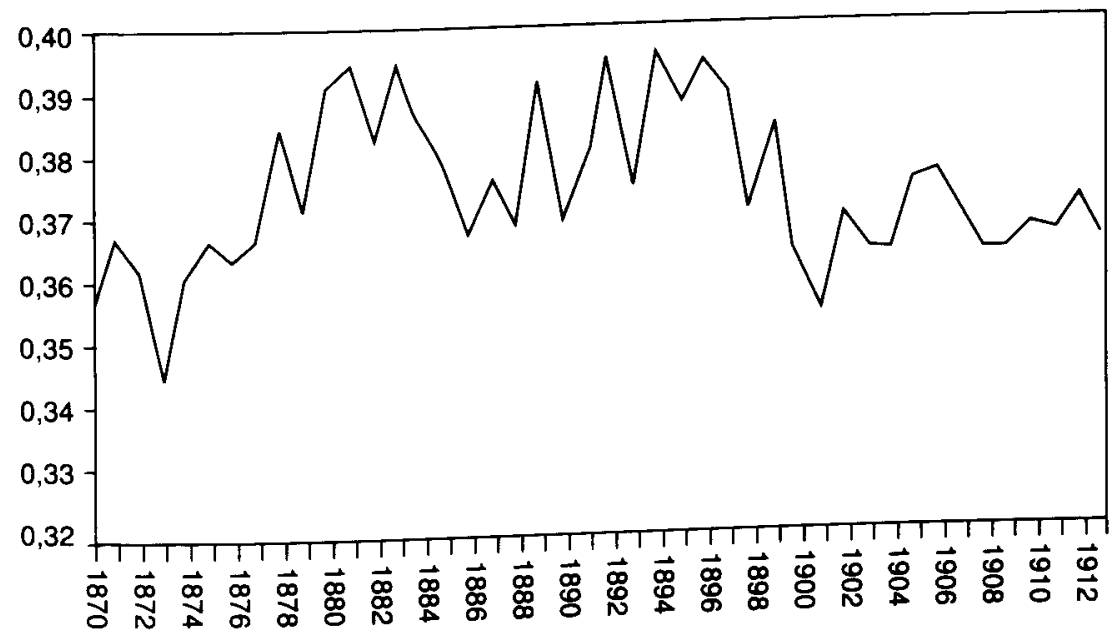

y los ricos en 1870, con un PIB per cápita relativo superior al 110 por 100 de la media (Bélgica, Holanda y Gran Bretaña).

Los resultados de este ejercicio no son demasiado esperanzadores. Como se observa en el Gráfico 2, en el que se presenta la desviación estándar del PIB per cápita dentro de estos grupos, no aparece patrón de convergencia alguno entre los países que los componen. Sólo en el caso de los países más pobres se identifica una reducción en la dispersión entre 1880 y 1900 , pero ésta se desvanece a lo largo de la primera década del siglo Xx. La desviación final es similar a la hallada al inicio del período.

Por lo tanto, esta división de la muestra tampoco nos sirve para encontrar las raíces de la ausencia de convergencia a nivel agregado. En este sentido, en el Gráfico 3 ofrecemos la descomposición de la evolución de la desviación estándar de la totalidad de la muestra en dos conceptos: la evolución de la dispersión en el seno de cada grupo (desviación intragrupos) y la de la dispersión existente entre los grupos (desviación intergrupos). Como podemos comprobar, los dos componentes de la desviación total mantienen una gran constancia a lo largo del período.

Sin embargo, el análisis comparativo de los niveles medios de PIB per cápita en cada uno de estos grupos nos ofrece una primera clave para 


\section{GRÁFICO 2}

Convergencia $\sigma$ por grupos de paises: 1870-1913

(desviación estándar PIB per cápita)

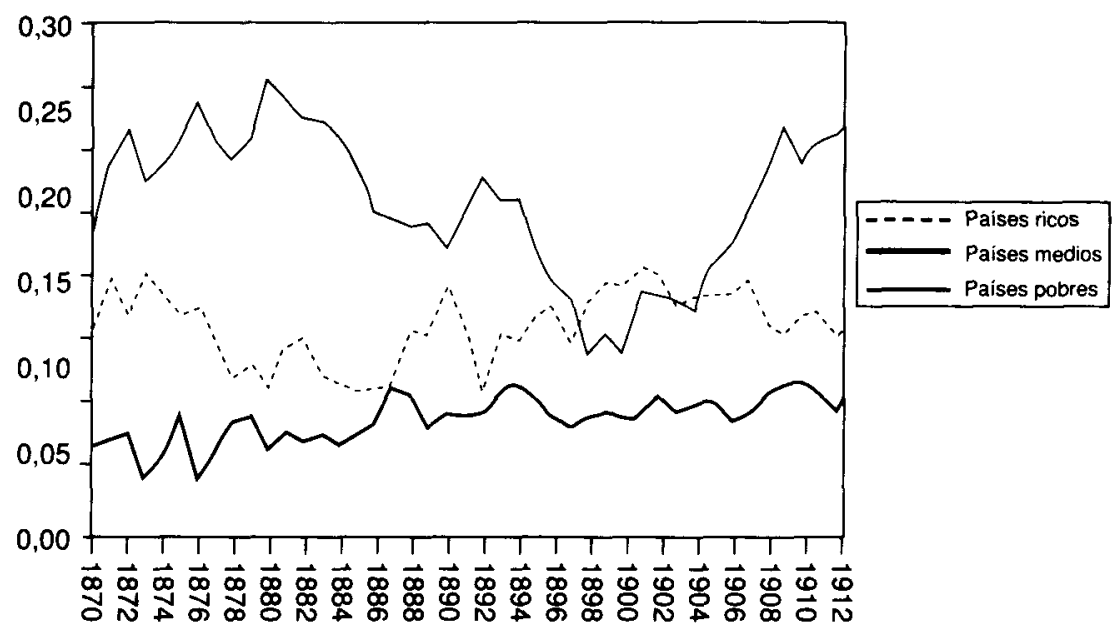

\section{GRÁFICO 3}

Convergencia o por grupos de paises: 1870-1913

(desviación estándar PIB per cápita)

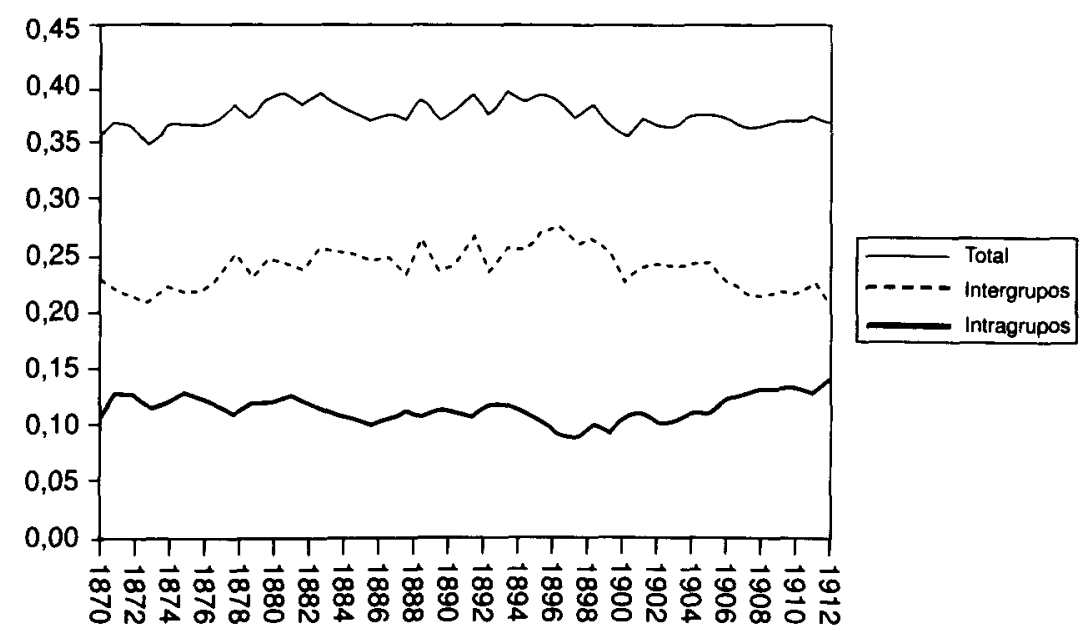


la caracterización de lo acontecido en las economías europeas durante estos años. Como se observa en el Gráfico 4, el nivel medio de PIB per cápita de los países intermedios se está aproximando al del grupo de los países ricos y separando del de los países pobres. La distancia entre estos dos últimos grupos, por contra, permanece constante.

\section{GRÁFICO 4 \\ Niveles medios de PIB per cápita por grupos \\ (logaritmos)}

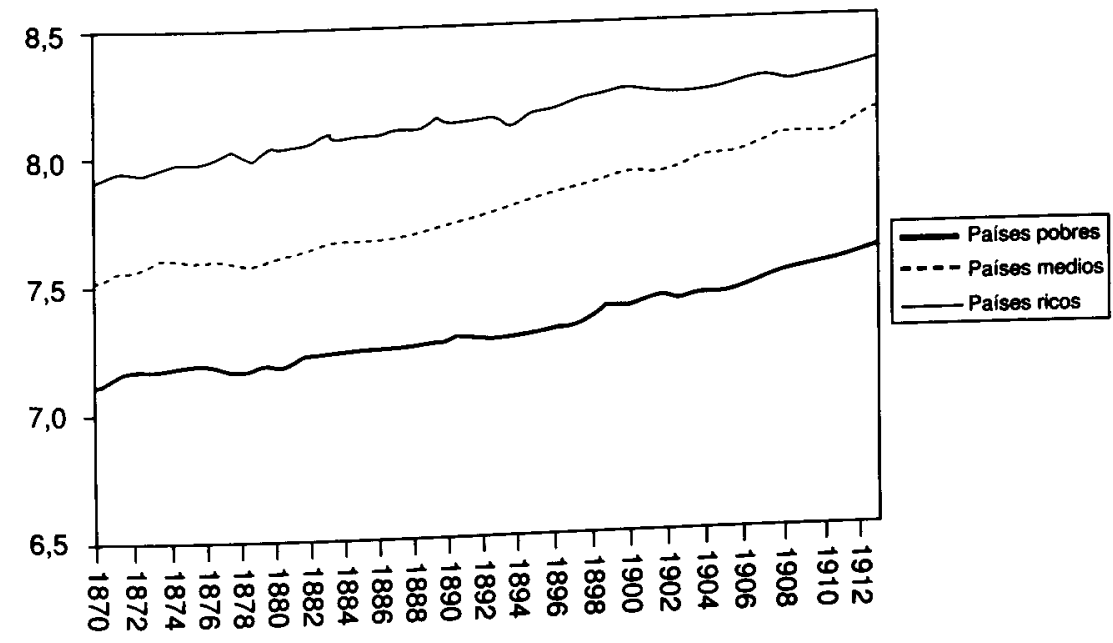

Esta evidencia sugiere la posibilidad de que se esté dando un proceso de $\sigma$-convergencia entre los países con PIB per cápita medio y alto, pero que éste no afecte a los países de renta más baja. Ésta es la hipótesis sobre la que vamos a concentrar nuestra atención a partir de ahora. Para ello, ahora dividimos la muestra en dos grupos. El primero sigue agrupando a los países de renta más baja. El segundo incluye los grupos de renta media y alta definidos en las líneas previas. Al descomponer la desviación total en la existente entre estos dos grupos y la que se da en el interior de los mismos, que presentamos en el Gráfico 5, observamos cómo se manifiesta una creciente homogeneización en el grupo de los países privilegiados. La $\sigma$-convergencia es un proceso exclusivo de los países de renta media y alta. Ahora bien, el sostenimiento de la desviación estándar 


\section{GRÁFICO 5}

Convergencia $\sigma$ por grupos: $1870-1913$

(desviación estándar PIB per cápita)

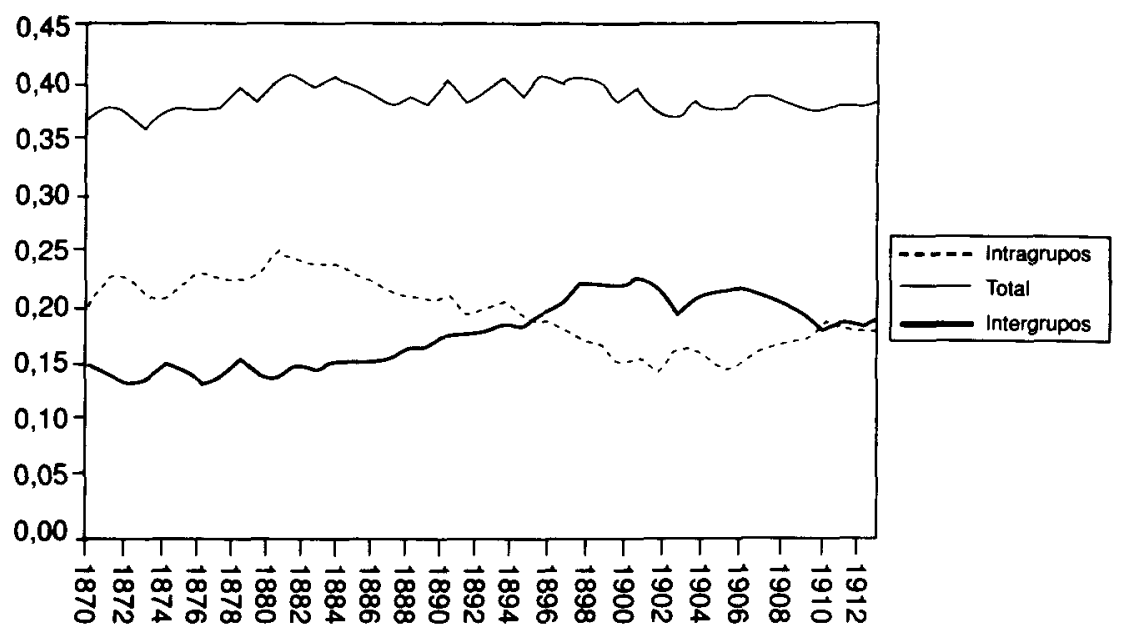

total es el resultado de la reducción en la dispersión media intragrupos y del aumento de la dispersión media entregrupos. La distancia existente entre los niveles medios de renta de un grupo y otro se mantiene o incluso presenta una ligera tendencia a la ampliación.

Para completar el estudio de la convergencia en Europa durante este período, proponemos la realización de algunos ejercicios de contraste de la existencia de convergencia $\beta^{35}$. La nueva base de datos permite contrastar esta hipótesis, aunque, del estudio previo, podríamos inferir que la evolución de los niveles de PIB per cápita de los países analizados no responde a este esquema de comportamiento, cuanto menos en su versión absoluta. En efecto, el hecho queda confirmado cuando observamos los resultados ofrecidos en la Tabla $1^{36}$.

${ }^{35}$ En este caso, la base de datos es un panel compuesto por 117 observaciones, que se obtienen al dividir las series nacionales de PIB per cápita en 9 tramos quinquenales. Con ello, disponemos de 9 datos de PIB per cápita de partida y de tasas de crecimiento del mismo para cada uno de los países analizados. Los períodos en los que se descompone la muestra son: $1870-74,75-79,80-84,85-89,90-94,95-99,1900-04,05-09,10-13$, por lo que el último corte temporal analizado sólo comprende cuatro años.

${ }^{36}$ En la forma estimada, los datos de PIB per cápita están expresados en diferencias 


\section{TABLA 1}

Convergencia $B$ absoluta

\begin{tabular}{|c|c|c|}
\hline \multicolumn{3}{|l|}{ Variable dependiense $\left(y_{i T}-y_{i 0}\right) / T$} \\
\hline Variable & Coeficiente & Estadístico \\
\hline $\begin{array}{l}y_{i 0} \\
n=117\end{array}$ & $\begin{array}{c}0,001 \\
\mathrm{R}^{2} \stackrel{0,002}{ }\end{array}$ & $-0,48$ \\
\hline
\end{tabular}

Nota. Estimación de la ecuación (6), por MCO, a partir de la base de datos descrita.

Los países situados en los puntos inferiores de la distribución tienden a crecer, como media, de forma más rápida que los más ricos, pero el ritmo al que recortan distancias es muy bajo (el coeficiente obtenido supondría un velocidad de convergencia del 0,1 por 100). Además, el coeficiente que relaciona ambas variables no es, considerando los niveles de confianza habituales, significativamente distinto de cero. La evidencia no permite aceptar la existencia de $\boldsymbol{\beta}$ convergencia absoluta.

En cualquier caso, como se ha apuntado en la sección 3, tampoco cabía esperar la aceptación de este tipo de comportamiento en una muestra compuesta por un grupo tan heterogéneo de países. No obstante, dado que el número de observaciones con las que trabajamos en este estudio lo permite, se ha considerado empíricamente la existencia de $\beta$-convergencia condicionada, es decir, a estados estacionarios distintos para cada uno de los países. De esta forma podremos comprobar el cumplimiento de la hipótesis de convergencia a largo plazo que se deriva de las formulaciones neoclásicas una vez se considera la posibilidad de que los niveles de renta a los que se acercan tendencialmente las distintas economías pueden diferir en función de cuáles sean las tasas de inversión y de crecimiento de la población propias de cada país. Para contrastar esta hipótesis hemos estimado un modelo de efectos nacionales fijos sobre una muestra de datos de panel ${ }^{37}$.

logarítmicas respecto a la media muestral. Este procedimiento es el propuesto por Marcet (1994), que corrige ligeramente al propuesto por Barro y Sala-i-Martin (1990) que ha sido presentado en la ecuación (1) y es apropiado para el estudio de la $\beta$-convergencia en muestras con datos de panel. En particular, la forma funcional estimada es:

$$
\left(y_{i T}-y_{i o}\right) / T=\alpha-\beta_{i o} y_{i o}+\varepsilon_{i}
$$

${ }^{37}$ En este caso se considera la posible existencia de estados estacionarios distintos 
TABLA 2

Convergencia $\beta$ condicionada

\begin{tabular}{|c|c|c|}
\hline \multicolumn{3}{|l|}{ Variable dependiente $\left(y_{i T}-y_{i 0}\right) / T$} \\
\hline Variables Explicativas & Coeficiente & Estadistico $t$ \\
\hline …........................ & 0,046 & 3,21 \\
\hline Gran Bretaña .......................... & 0,023 & 2,80 \\
\hline 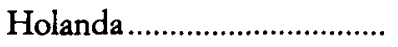 & 0,014 & 2,56 \\
\hline 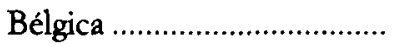 & 0,013 & 2,36 \\
\hline 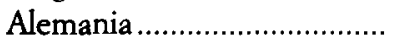 & 0,008 & 2,68 \\
\hline 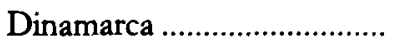 & 0,005 & 1,76 \\
\hline 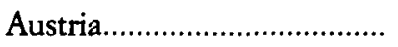 & 0,004 & 1,67 \\
\hline 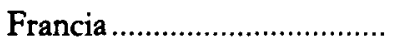 & 0,003 & 1,29 \\
\hline 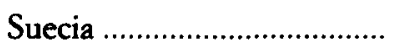 & $-0,001$ & $-0,62$ \\
\hline 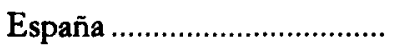 & $-0,014$ & $-2,73$ \\
\hline 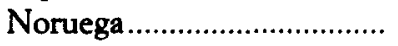 & $-0,016$ & $-2,75$ \\
\hline 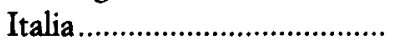 & $-0,017$ & $-3,12$ \\
\hline 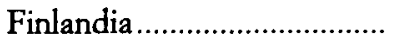 & $-0,023$ & $-2,96$ \\
\hline 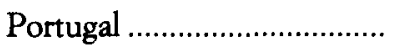 & $-0,038$ & $-3,54$ \\
\hline $\mathrm{n}=117 \ldots \ldots \ldots \ldots \ldots \ldots \ldots \ldots \ldots \ldots \ldots \ldots \ldots$ & $\mathrm{R}^{2}=0,15$ & $\mathrm{SE}=0,0082$ \\
\hline
\end{tabular}

Nota. Estimación de la ecuación (7) a partir de la base de datos descrita. Estimación por MCO con datos de panel.

La realización de este ejercicio, cuyos primeros resultados se ofrecen en la Tabla 2, nos permite señalar un conjunto de ideas que entendemos relevantes. En el caso de considerar la presencia de efectos nacionales que conduzcan a la existencia de estados estacionarios distintos entre los países, el análisis econométrico avala la hipótesis de $\beta$ convergencia. Además, el valor que vincula los niveles iniciales de renta con los ritmos posteriores de crecimiento, que nos aproxima a la velocidad a la que cada

para las diferentes economías. Para captar esta posibilidad se introducen variables ficticias para cada uno de los países en la función estimada, que será del tipo:

$$
\left(y_{i T}-y_{i 0}\right) / T=\beta_{i}\left(\Sigma i D u m m y_{i}-y_{i 0}\right)+\varepsilon_{i}
$$

este modelo, como se comentaba anteriormente, equivale a la estimación de un modelo de «efectos fijos». En Arellano y Bover (1990) se puede encontrar una descripción detallada de estos modelos de datos de panel. 
economía se acerca a su estado estacionario, es muy elevado, del orden del 4,6 por $100^{38}$.

Por otra parte, cabe destacar que las variables ficticias que absorben la presencia de efectos nacionales son significativas en la mayor parte de casos, lo que se debe entender como un indicio de su persistencia. Analizando los valores y significatividad individual de estas variables ficticias, obtenemos un resultado adicional. En la Tabla 2 hemos ordenado los distintos países en función del grupo en el que quedaban inscritos en el estudio de la $\sigma$-convergencia. En este caso se comprueba que los países de renta alta en 1870 (Gran Bretaña, Holanda y Bélgica) muestran unos efectos fijos positivos y significativos. Los de renta baja (España, Italia, Portugal, Finlandia y Noruega) experimentan la situación opuesta. Los coeficientes que nos muestran sus efectos nacionales fijos son significativos, pero en este caso, negativos. El grupo intermedio (Austria, Dinamarca, Alemania, Suecia y Francia) ofrece una situación menos definida. En la mayor parte de casos los efectos fijos son positivos (la excepción sería Suecia), pero la significatividad de los estimadores sólo sería satisfactoria en el caso de Alemania.

El ejercicio permite una extensión inmediata, considerar los valores estimados de estos efectos nacionales para calcular los estados estacionarios hacia los que se acerca, de forma tendencial, cada una de las economías ${ }^{39}$. Su cálculo nos permitirá comparar los valores estimados con la situación real de las economías en 1913. El Gráfico 6 presenta los valores de una y otra variables, ordenando los países de menor a mayor según su posición relativa estimada de equilibrio. Se puede destacar que, como media, las economías se sitúan en 1913 en una posición cercana a la que suponen los estados estacionarios. Además, se apunta el posicionamiento de los países en dos grupos bien definidos, con niveles de equilibrio próximos entre sí. Uno cercano al 60-70 por 100 de la media muestral y otro un 20-30 por 100 por encima de ésta.

Por lo tanto, del análisis empírico de la convergencia basado en datos de corte transversal, podemos concluir que se acepta la existencia de $\beta$-convergencia condicionada a estados estacionarios que son distintos y per-

${ }^{38}$ Cabe recordar que el valor del coeficiente alcanzado a través de estas estimaciones con datos de panel puede estar sesgado al alza al recoger la velocidad con la que se regresa al valor medio tras alguna perturbación cíclica. De la Fuente (1998), p. 14.

39 Sin embargo, De la Fuente (1998), p. 14, nos recuerda que los valores de estado estacionario estimados a través de modelos de efectos fijos con datos de panel no constituyen un indicador definitivo de los valores de equilibrio de largo plazo a los que tienden las economías. No obstante, podemos tomarlos como orientativos de los mismos. 


\section{GRÁFICO 6}

PIB per cápita relativo estimado y real en 1913

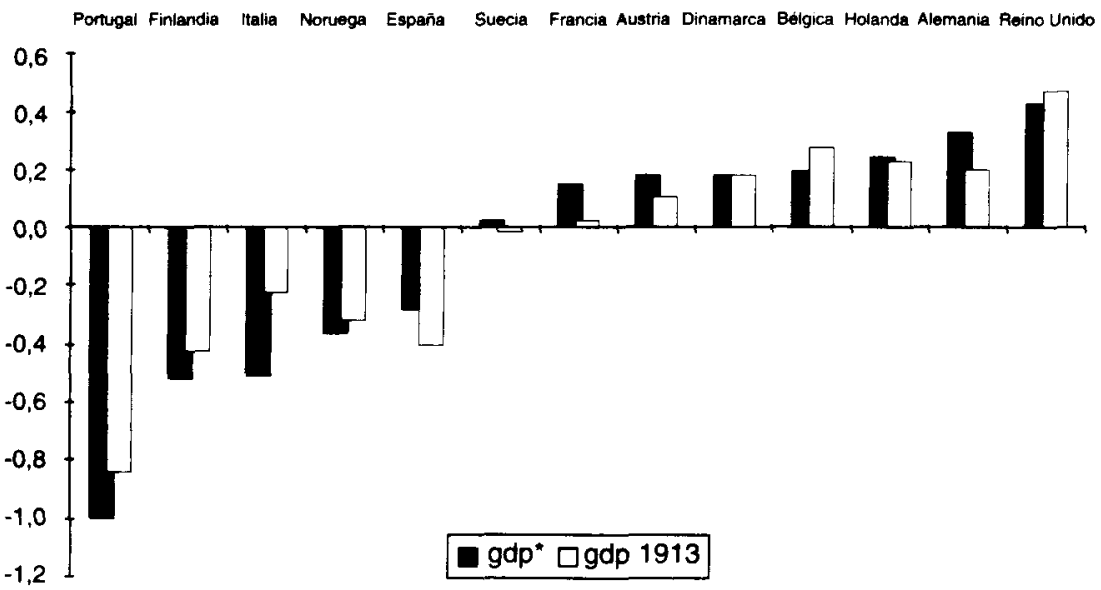

Nota: gdp* es el PIB per cápita relativo estimado de estado estacionario para cada uno de los países. gdp1913 se corresponde con el PIB per cápita relativo real en ese fecha.

sistentes para cada uno de los países. Por ello, la evolución de la economía promedio puede estar ocultando experiencias individuales o de subgrupos muy diversas, como parece derivarse del análisis de la $\sigma$-convergencia o de los valores estimados para los efectos fijos a los que están sujetas las distintas economías. En estas circunstancias, parece especialmente relevante estudiar las experiencias individuales mediante la metodología empírica de la convergencia con datos de serie temporal. Éste es el objeto de las próximas páginas.

\subsection{Análisis de la convergencia con datos de serie temporal}

En esta sección se comentan los resultados obtenidos al estudiar los procesos de convergencia entre los países europeos en el período 1870-1913 utilizando el enfoque de series temporales anteriormente descrito, con el objeto de completar la evidencia sugerida anteriormente. Este estudio requiere, en primer lugar, efectuar un análisis del orden de integrabilidad de las series de producto per cápita de los países considerados, así como 
de la media de los países de renta alta y de renta media. Para ello se han utilizado los contrastes de Dickey y Fuller Aumentado, Phillips y Perron y, finalmente, la propuesta de Kwiatkowski, Phillips, Schmidt y Shin (1992), que considera la hipótesis nula de estacionariedad en lugar de la de raíz unitaria, como es el caso de los dos anteriores. La evidencia obtenida permite aceptar en todos los casos la existencia de una raíz unitaria para las series de PIB per cápita de los países analizados y de las agregaciones efectuadas, y la estacionariedad de las primeras diferencias de las mismas. Es decir, las series de producto per cápita utilizadas en este estudio son I(1), lo que hace posible encontrar relaciones de cointegración para cualquier par de series.

Al utilizar el enfoque de series temporales es preciso seleccionar una economía que detente la posición de líder, para realizar un análisis de la relación que mantienen el resto de economías con ella. En este trabajo se ha establecido, en primer lugar, que la economía líder es la media de los países considerados ricos (Bélgica, Holanda y Reino Unido) y se ha estudiado si cada uno de los países de renta media (Austria, Dinamarca, Francia, Alemania y Suecia) y de los pobres (Italia, Noruega, Finlandia, España y Portugal) presenta un proceso de convergencia absoluta o de catching-up con la media de los países ricos. En segundo lugar, se ha considerado como economía líder a la media de los países con una renta intermedia en 1870 , y se han estudiado las relaciones que mantiene con cada uno de los países considerados pobres.

En la Tabla 3 se presentan los resultados obtenidos al utilizar la media de los países ricos como economía líder. Se observa cómo en cuatro casos se puede rechazar la existencia de una raíz unitaria y, por tanto, la hipótesis de no convergencia. A pesar de ello, si se establece un nivel de significación $\alpha=0,05$, únicamente en los casos de Alemania y Francia se puede rechazar la hipótesis nula de no convergencia. En el caso de Alemania, el modelo que resulta relevante apunta a una trayectoria de convergencia a largo plazo, mientras que en el caso de Francia es más difícil escoger entre las dos opciones de convergencia, aunque también nos inclinamos por la hipótesis de convergencia absoluta, puesto que con un nivel de significación del 5 por 100 no es posible rechazar la no presencia de una tendencia determinista en la serie que recoge la diferencia en el PIB per cápita de Francia y el medio de los países considerados ricos. En el caso de Austria podría aceptarse la hipótesis de convergencia absoluta, mientras que en el caso de Finlandia se observa un proceso de catching-up, aunque en estos dos 


\section{TABLA 3}

Hipótesis de convergencia. Economia líder: media países ricos

\begin{tabular}{|c|c|c|c|}
\hline & $\begin{array}{c}\text { Nivel } \\
\text { Modelo IOM-I }\end{array}$ & $\begin{array}{c}\text { Tendencia } \\
\text { Modelo AOM-II }\end{array}$ & $\begin{array}{l}\text { Nivel-tendencia } \\
\text { Modelo IOM-III }\end{array}$ \\
\hline \multicolumn{4}{|l|}{ Renta Media } \\
\hline 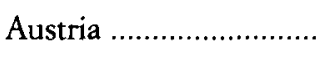 & $\begin{array}{l}-5,11^{b} \\
(1906)\end{array}$ & $\begin{array}{l}-3,61 \\
(1873)\end{array}$ & $\begin{array}{l}-4,87 \\
(1906)\end{array}$ \\
\hline Dinamarca ........................ & $\begin{array}{l}-4,18 \\
(1902)\end{array}$ & $\begin{array}{l}-4,06 \\
(1889)\end{array}$ & $\begin{array}{l}-4,28 \\
(1893)\end{array}$ \\
\hline . & $\begin{array}{l}-5,28^{a} \\
(1878)\end{array}$ & $\begin{array}{l}-4,74^{b} \\
(1886)\end{array}$ & $\begin{array}{l}-5,43^{b} \\
(1875)\end{array}$ \\
\hline Alemania ............................ & $\begin{array}{l}-5,76^{\mathrm{a}} \\
(1879)\end{array}$ & $\begin{array}{l}-4,14 \\
(1887)\end{array}$ & $\begin{array}{l}-4,66 \\
(1880)\end{array}$ \\
\hline Suecia ................................ & $\begin{array}{l}-4,44 \\
(1885)\end{array}$ & $\begin{array}{l}-4,21 \\
(1885)\end{array}$ & $\begin{array}{l}-4,64 \\
(1886)\end{array}$ \\
\hline \multicolumn{4}{|l|}{ Renta Baja } \\
\hline 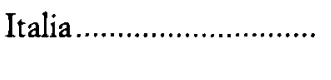 & $\begin{array}{l}-2,39 \\
(1888)\end{array}$ & $\begin{array}{l}-4,24 \\
(1899)\end{array}$ & $\begin{array}{l}-5,08 \\
(1895)\end{array}$ \\
\hline 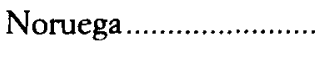 & $\begin{array}{l}-3,61 \\
(1907)\end{array}$ & $\begin{array}{l}-3,38 \\
(1905)\end{array}$ & $\begin{array}{l}-3,39 \\
(1903)\end{array}$ \\
\hline 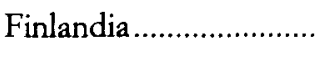 & $\begin{array}{l}-3,16 \\
(1877)\end{array}$ & $\begin{array}{l}-4,64^{b} \\
(1899)\end{array}$ & $\begin{array}{l}-5,17 \\
(1890)\end{array}$ \\
\hline Portugal .......................... & $\begin{array}{l}-4,22 \\
(1904)\end{array}$ & $\begin{array}{l}-4,39 \\
(1903)\end{array}$ & $\begin{array}{l}-4,82 \\
(1896)\end{array}$ \\
\hline 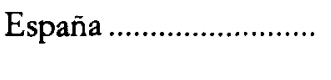 & $\begin{array}{l}-4,10 \\
(1893)\end{array}$ & $\begin{array}{l}-3,99 \\
(1903)\end{array}$ & $\begin{array}{l}-4,52 \\
(1893)\end{array}$ \\
\hline
\end{tabular}

Nota. Los valores críticos proceden de Perron (1994).

Entre paréntesis aparece el punto de ruptura (TB) determinado endógenamente.

${ }^{a} \mathrm{y}^{\mathrm{b}}$ significan rechazo de la hipótesis nula de no convergencia con un nivel de significación $\alpha=0,05$ y $\alpha=0,10$, respectivamente. Se han señalado en negrita los casos en que se rechaza la hipótesis de no convergencia.

últimos casos la hipótesis de no convergencia únicamente se puede rechazar si se rebaja considerablemente el nivel de significación.

Por lo tanto, los resultados obtenidos sugieren que los países con renta media en el instante inicial del período analizado presentan un proceso de convergencia (Alemania, Francia y Austria, aunque en este último caso la evidencia es menos concluyente) o están próximos al rechazo de la hipótesis de no convergencia (Dinamarca y Suecia), mientras que los de renta 
baja, con la excepción de Finlandia y con la salvedad expresada anteriormente, no presentan un proceso de convergencia con los países de renta alta.

\section{TABLA 4}

Hipótesis de convergencia. Economia lider: media países con renta media

\begin{tabular}{|c|c|c|c|}
\hline Renta Baja & $\begin{array}{c}\text { Nivel } \\
\text { Modelo IOM-I }\end{array}$ & $\begin{array}{c}\text { Tendencia } \\
\text { Modelo AOM-II }\end{array}$ & $\begin{array}{l}\text { Nivel-tendencia } \\
\text { Modelo IOM-III }\end{array}$ \\
\hline 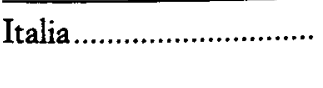 & $\begin{array}{l}-3,16 \\
(1906)\end{array}$ & $\begin{array}{l}-3,87 \\
(1898)\end{array}$ & $\begin{array}{l}-4,57 \\
(1893)\end{array}$ \\
\hline Noruega ........................... & $\begin{array}{r}-4,50 \\
(1909)\end{array}$ & $\begin{array}{l}-4,75^{b} \\
(1906)\end{array}$ & $\begin{array}{l}-4,56 \\
(1906)\end{array}$ \\
\hline Finlandia ........................ & $\begin{array}{l}-5,37^{b} \\
(1880)\end{array}$ & $\begin{array}{l}-5,31^{b} \\
(1892)\end{array}$ & $\begin{array}{l}-5,52^{b} \\
(1894)\end{array}$ \\
\hline Portugal ........................... & $\begin{array}{r}-3,82 \\
(1908)\end{array}$ & $\begin{array}{l}-4,63^{b} \\
(1902)\end{array}$ & $\begin{array}{l}-4,44 \\
(1899)\end{array}$ \\
\hline 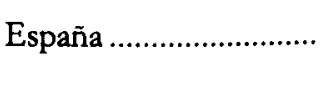 & $\begin{array}{l}-4,78 \\
(1894)\end{array}$ & $\begin{array}{r}-3,83 \\
(1880)\end{array}$ & $\begin{array}{l}-4,38 \\
(1887)\end{array}$ \\
\hline
\end{tabular}

Nota. Los valores críticos proceden de Perron (1994).

Entre paréntesis aparece el punto de ruptura (TB) determinado endógenamente.

$\mathrm{a}^{\mathrm{b}}$ significan rechazo de la hipótesis nula de no convergencia con un nivel de significación $\alpha=0,05$ y $\alpha=0,10$, respectivamente. Se han señalado en negrita los casos en que se rechaza la hipótesis de no convergencia.

En la Tabla 4 se presentan los resultados obtenidos al considerar como economía líder el promedio de renta de los países de renta media. En este caso se ha estudiado si existe un proceso de convergencia a largo plazo entre esta economía y los países considerados pobres. La evidencia obtenida sugiere que existe un proceso de catching-up en el caso de Finlandia y de manera menos clara en los casos de Noruega y de Portugal ${ }^{40}$, mientras que en el resto de países - Italia y España - no es posible rechazar la hipótesis de no convergencia.

${ }^{40}$ Los resultados obtenidos en el análisis de la economía portuguesa no se corresponden con la percepción más extendida sobre el comportamiento agregado de esta economía durante los años 1870-1913, que sería la de atraso creciente. En este sentido, deberíamos apuntar que estos resultados pueden resentirse de la opción tomada en este estudio. Incorporar una serie de PIB per cápita portugués que no es estrictamente comparable con el resto de datos analizados. 


\section{CONCLUSIONES}

En las páginas previas hemos ofrecido una primera caracterización de la evolución del PIB per cápita relativo en Europa en el período previo a la I Guerra Mundial. Ésta confirma una serie de hechos estilizados que podemos resumir del siguiente modo. La dinámica de crecimiento comparado en Europa no vino caracterizada por el cumplimiento de la hipótesis de $\sigma$-convergencia. No se registró una reducción de la dispersión de los niveles de PIB per cápita entre los distintos países.

Los resultados del contraste de la hipótesis de $\beta$-convergencia no condicionada tampoco son satisfactorios. Aunque el signo del parámetro de convergencia estimado sea el esperado, ni su tamaño ni su significatividad nos permiten abogar por el cumplimiento de esta hipótesis. Como media, el ritmo de crecimiento de las economías no estuvo inversamente vinculado al nivel de PIB per cápita de partida. Los países más pobres no crecieron de forma más rápida que los ricos.

Sin embargo, una vez consideradas las posibles diferencias en los estados estacionarios mediante la estimación de un modelo que incorpora la existencia de efectos nacionales fijos, podemos aceptar el cumplimiento de la hipótesis de $\beta$-convergencia condicionada. Los parámetros que captan la dimensión de los efectos nacionales son muy significativos, lo que sería un indicio de su persistencia. Los valores de estos parámetros permiten realizar una primera aproximación a los valores de estado estacionario a los que se aproximan las distintas economías. De su análisis se deriva que el comportamiento promedio esconde la existencia de trayectorias muy dispares. En particular, se observa que los países parecen converger hacia dos niveles de renta per cápita distintos, uno cercano al 60-70 por 100 de la media muestral y otro un 20-30 por 100 por encima de esta media.

Para profundizar en el estudio de las experiencias individuales se ha realizado un análisis empírico de la convergencia utilizando metodología de series temporales. Éste muestra cómo existe una mayor dinámica de acercamiento a los niveles de PIB per cápita de los países ricos en las experiencias propias de los países de renta media (Alemania, Francia o Austria). Sin embargo, revela que los países que parten de unos niveles de PIB per cápita más bajos no convergen con los países más ricos (sólo se rechaza la hipótesis de no convergencia en el caso de Finlandia y con un bajo nivel de significación).

Por lo tanto, del estudio se desprende que, a la hora de explicar las diferentes trayectorias relativas seguidas por las economías europeas, es 
necesario considerar la situación de partida en términos de PIB per cápita. Durante estos años, el proceso de convergencia en términos de PIB per cápita sólo fue factible en el caso de economías que partían de unos niveles de desarrollo relativo elevados.

\section{BIBLIOGRAFÍA}

ABRAmovirz, M. (1986): «Catching up, forging ahead and falling behind», Joumal of Economic History, núm. 46, pp. 385-406.

ARELlano, M., y Bover, O. (1990): «La econometría de datos de panel», Investigaciones Económicas, núm. 14, pp. 3-45.

ARROW, K. H. (1962): «The economic implications of Learning by Doing», Review of Economic Studies, núm. 29, pp. 155-173.

AZARIADIS, C., y DrazEN, A. (1990): «Threshold Externalities in Economic Development», Quarterly Joumal of Economics, núm. 90, pp. 501-526.

BANERJEE, A.; LUMSDANE, R. L., y STOCK, J. H. (1992): «Recursive and sequential tests of the unit -root and trend-break hypotheses: Theory and international evidence», Journal of Business and Economic Statistics, núm. 10, pp. 271-287.

Bardini, C.; Carreras, A., y Lains, P. (1995): «The national accounts for Italy, Spain and Portugal», Scandinavian Economic History Review, núm. 43, pp. 115-146.

BARRO, R. (1991): «Economic growth in a cross-section of countries», Quarterly Journal of Economics, núm. 106, pp. 407-443.

BARRO, R., y SALA-I-MARTTN, X. (1990): «Economic Growth and convergence across the United States», Working Paper NBER, núm. 3419, Cambridge, MA.

BAUMOL, W. J. (1986): «Productivity growth, convergence and welfare: what the long-run data show", American Economic Review, núm. 76, pp. 1072-1085.

Baumol, W. J., y Wol., E. N. (1988): «Productivity Growth, Convergence, and Welfare: Reply», American Economic Review, núm. 78, pp. 1155-1159.

BEN-DAVID, D., y PAPELL, D. H. (2000): «Some evidence on the continuity of the growth process among the G7 countries», Economic Inquiry, núm. 38, pp. 320-330.

BERNARD, A., y DURLAUF, S. (1995): «Convergence in international output», Journal of Applied Econometrics, núm. 10, pp. 97-108.

BERNARD, A., y DURLAUF, S. (1996): «Interpreting tests of convergence hypothesis», Joumal of Econometrics, núm. 71, pp. 161-173.

BROADBERRY, S. N. (1996): «Convergence: what the historical record shows», en B. VAN ARK y N. F. R. CRAFTs, Quantitative aspects of Postwar European Economic Growth, Cambridge, CEPR-Cambridge University Press, pp. 327-346.

CAMPBell, J., y Perron, P. (1991): «Pitfalls and oportunities: What macroeconomists should know about unit roots», NBER Macroeconomics Annual, pp. 141-201.

CARLINO, G., y MILLS, L. (1993): «Are US regional income converging?», Joumal of Monetary Economics, núm. 32, pp. 335-346. 
Cuadrado Roura, J. R.; Mancha, T., y Garrido, R. (dirs.) (1998): Convergencia regional en España: Hechos, tendencias y perspectivas, Madrid, Fundación Argentaria y Visor.

CRAFTS, N. F. R. (1998): «Forging ahead and falling behind: the rise and relative decline of the first industrial nation», Joumal of Economic Perspectives, núm. 12, pp. 193-211.

DE LA FUENTE, A. (1997): «The empirics of growth and convergence: a selective review», Joumal of Economic Dynamics and Control, núm. 21, pp. 23-73.

- (1998): «Algunas técnicas para el análisis de la convergencia con una aplicación a las regiones españolas», Documentos de Trabajo, D.98007, Ministerio de Economía y Hacienda, Madrid.

DuRLAUF, N. S. (1996): «A theory of persistent income inequality», Journal of Economic Growth, núm. 1, pp. 75-94.

GaLOR, O. (1996): «Convergence? Inferences from theoretical models», Economic Journal, núm. 106, pp. 1056-1069.

GalOR, O., y ZEIRA, J. (1993): «Income distribution and macroeconomics», Review of Economic Studies, núm. 60, pp. 35-52.

Grossman, G., y HelPMAN, E. (1991): Innovation and growth in the global economy, Cambridge, MA, MIT Press.

Kwiatkowski, D.; Phillips, P. C. B.; Schmidt, P., y SHIN, Y. (1992): «Testing the null of stationary against the alternative of a unit root», Journal of Econometrics, núm. 54, pp. 159-178.

LUCAS, R. (1988): «On the mechanics of economic development», Journal of Monetary Economics, núm. 22, pp. 3-42.

Maddison, A. (1982): Phases of capitalist development, Oxford, Oxford University Press.

- (1991): Dynamics forces in capitalist development: $A$ long run comparative view, Oxford, Oxford University Press.

- (1995): Monitoring the World Economy, Paris, OECD.

MANKT, N.; ROMER, D., y WEIL, D. (1992): «A contribution to the empirics of economic growth», Quarterly Journal of Economics, núm. 107, pp. 407-437.

MARCET, A. (1994): «Los pobres siguen siendo pobres: convergencia entre regiones y países, un análisis bayesiano con datos de panel», en J. M. ESTEBAN y X. VIVEs (dirs.), Crecimiento y convergencia regional en España y Europa, vol. II, Instituto de Análisis Económico, Barcelona.

MurPHY, K. M.; SChleIfer, A., y VISHNY, R. W. (1989): «Industrialization and tha Big Push», Quarterly Journal of Economics, núm. 106, pp. 503-530.

NG, S., y PERron, P. (1995): «Unit root tests in ARMA models with data dependent methods for the selection of the truncation lag», Joumal of the American Statistical Association, núm. 90, pp. 268-281.

O'Rourke, K., y Williamson, J. G. (1997): «Around the European periphery 1870-1913: Globalization, schooling and growth», European Review of Economic History, núm. 1, pp. 153-190.

OxIEY, L., y GrEasLey, D. (1995): «A time-series perspective on convergence: Australia, UK and USA since 1870», The Economic Record, núm. 71, pp. 259-270. 
Pallardó, V. J., y Esteve, V. (1997): «Convergencia real en la Unión Europea», Revista de Economia Aplicada, núm. 14, pp. 25-49.

PERRon, P. (1989): «The great crash, the oil price shock, and the unit root in time series regression», Econometrica, núm. 57, pp. 1361-1401.

- (1994): «Further evidence on breaking trend functions in macroeconomic variables», Working Paper, núm. 2594, CRDE, Université de Montréal.

Prados de la Escosura, L. (1995): Spain's Gross Domestic Product, 1850-1990: Quantitative Conjectures, Madrid, Universidad Carlos III, Documentos de Trabajo.

Prados de la Escosura, L.; Daban, T., y Sanz, J. C. (1993): «De te fabula narratur? Growth, Structural Change and Convergence in Europe, 19th-20th Centuries», Working Paper D-93009, Dirección General de Planificación, Ministerio de Economía y Hacienda, Madrid.

Prados de la Escosura, L., y SANZ, I. (1998): «Historical comparisons of Income: A Short-cut approach», en B. van ARK, E. BUYST y J. L. VAN ZANDEN (org.), Historical benchmark comparisons of output and productivity, B10 Proceedings Twelfth International Economic History Congress, Madrid.

QUAH, D. (1993a): «Empirical cross-section dynamics in economic growth», European Economic Review, núm. 37, pp. 426-434.

- (1993b): «Galton's Fallacy and test of the convergence hypothesis», Scandinavian Joumal of Economics, núm. 95, pp. 427-443.

- (1996a): «Regional convergence clusters across Europe», European Economic Review, núm. 40, pp. 951-958.

- (1996b): «Twin peaks: Growth and convergence in models of distribution dynamics», Economic Journal, 106, pp. 1045-1055.

- (1997): «Empirics for growth and distribution: Stratification, polarization, and convergence clubs», Discussion Paper, núm. 324, LSE Economics Department.

RAPPOPORT, P., y ReIChlin, L. (1989): «Segmented trends and non-stationary time series», Economic Journal, núm. 99, pp. 168-177.

Romer, P. M. (1986): «Increasing Returns and long-run growth», Joumal of Political Economics, 94, 5, pp. 1002-1037.

SALA-I-MARTin, X. (1996): «The classical approach to convergence analysis», Economic Journal, núm. 106, pp. 1019-1036.

SHELl, K. (ed.) (1967): Essays on the Theory of Optimal Economic Growth, Cambridge, MTT Press.

Solow, R. (1956): «A contribution to the theory of Economic Growth», Quarterly Journal of Economics, núm. 70, pp. 65-94.

TAYLOR, A. M. (1999): «Sources of convergence in the late nineteenth century», European Economic Review, núm. 43, pp. 1621-1645.

TORTEllA, G. (1994): «Patterns of economic retardation and recovery in South-Western Europe in the Nineteenth and Twentieth Centuries», Economic History Review, núm. 47, pp. 1-21.

Williamson, J. G. (1995): «The Evolution of Global Labor Markets Since 1830: Background Evidence and Hypotheses», Explorations in Economic History, núm. 32, pp. 141-196. 
- (1996): «Globalization, convergence and history», Journal of Economic History, núm. 56, pp. 277-306.

ZINOT, E., y ANDREWS, D. W. K. (1992): «Further evidence on the great crash, the oil price and the unit root hypothesis», Journal of Business and Economic Statistics, núm. 10, pp. 251-270. 